\title{
SUPPORT, CONVERGENCE, AND DIFFERENTIABILITY PROPERTIES OF GENERALIZED CONVEX FUNCTIONS
}

\author{
JOHN W. GREEN
}

1. Introduction. The notion of sub- $F$ functions has been introduced by Beckenbach [1] and studied further by Peixoto $[5 ; 6 ; 7]$ and Bonsall [3]. Briefly, these may be described as follows: In an interval I: $a<x<b$, there exists a two parameter family $\mathcal{F}$ of continuous functions $F$ such that given $\left(x_{1}, y_{1}\right)$ and $\left(x_{2}, y_{2}\right)$, with $x_{1}$ and $x_{2}$ in $I$ and $x_{1} \neq x_{2}$, there is exactly one function $F$ of $\mathcal{f}$ such that $F\left(x_{1}\right)=y_{1}$ and $F\left(x_{2}\right)=y_{2}$. A function $f$ is sub- $F$ provided if $f$ and $F$ agree at $x_{1}$ and $x_{2}$ then $f \leqq F$ between $x_{1}$ and $x_{2}$.

Beckenbach showed that the sub- $F$ functions possess a number of properties analogous to those of convex functions, and we list below such of his results as will be used later in this note. It will be convenient to speak of the functions of $\mathcal{F}$ as $F$-lines, and it is clear what is meant by saying that two points determine an $F$-line. All $x$ appearing will belong to $I$, although it may not be so stated in each case. The following statements are given informally, but their exact content is easy to see.

B1. If two F-lines have a point in common and are not identical, they cross at that point.

B2. If two sequences of points tend to limits with different abscissas, the F-lines determined by the successive pairs of points of the sequences tend to the F-line determined by the limit points, uniformly in every compact subinterval of $I$.

B3. If $f$ is sub-F and agrees with $F$ at $x_{1}$ and $x_{2}$. then outside the interval $\left(x_{1}, x_{2}\right), f \geqq F$.

B4. $A$ sub-F function is continuous.

An $F$-line is a support of $f$ at $x_{0}$ provided $F \leqq f$ in $I$ and $F\left(x_{0}\right)=f\left(x_{0}\right)$. Peixoto [5] obtained among others the following results.

P1. A sub-F function has a support at each point $x_{0}$. If the support is not unique, there are two extreme supports, $F^{+}$and $F^{-}$, crossing at $x_{0}$ and such that (a) every support to $f$ at $x_{0}$ lies between $F^{+}$and $F^{-}$, and (b) every F-line which lies between $F^{+}$and $F^{-}$supports $f$ at $x_{0}$.

The support $F^{+}$is obtained by passing the $F$-line through $\left(x_{0}, f\left(x_{0}\right)\right)$ and $\left(x_{1}, f\left(x_{1}\right)\right)$, where $x_{1}>x_{0}$, and allowing $x_{1}$ to approach $x_{0}$.

P2. If almost everywhere in I each F-line has finite lower derivatives, then a sub-F function has a derivative almost everywhere.

Received by the editors June 4, 1952. 
P3. If all $F$ passing through $\left(x_{0}, y_{0}\right)$ have a uniformly bounded second derivative in a neighborhood of $x_{0}$, then a sub-F function has a derivative except at countably many points.

In the present note we shall obtain further supporting and differentiability properties partly overlapping and partly extending those obtained by Peixoto. The author has had occasion to make analytic use of a certain class of sub- $F$ functions, namely the sub-sine functions (see $\$ 4$ ), and the hypotheses placed on $\mathcal{F}$ in this note are suggested by that use. We shall also obtain a pair of theorems on the convergence of sequences of sub- $F$ functions.

The families considered by Bonsall are somewhat less general than those of the present paper, being the solutions of certain second order differential equations. Also he obtains a stronger result concerning the one item where there is essential overlap in subject matter between the two papers; namely, he shows that his sub-functions have second derivatives almost everywhere. Our Theorem 5 is related to his corollary to Theorem 1, and our Theorem 3 to his Theorem 2 . In our application, the family involved is of the type considered by Bonsall, and the result could equally have been deduced from his work.

\section{Support and convergence.}

Lemma 1. Let $F_{0}$ and $F_{1}$ support the sub-F function $f$ at $x_{0}$ and $x_{1}$ respectively, where $x_{0}<x_{1}$. Then for $x>x_{1}$, we have $F_{0} \leqq F_{1}$.

Suppose that for $x_{2}>x_{1}$ we have $F_{1}\left(x_{2}\right)<F_{0}\left(x_{2}\right)$. Since $F_{1}\left(x_{1}\right)=f\left(x_{1}\right)$ $\geqq F_{0}\left(x_{1}\right), F_{1}$ and $F_{0}$ must cross at $x_{1}$ or to the right of it. In either case, to the left of $x_{1}, F_{1}>F_{0}$; in particular, $F_{1}\left(x_{0}\right)>F_{0}\left(x_{0}\right)=f\left(x_{0}\right)$, which contradicts the fact that $F_{1}$ supports $f$ at $x_{1}$.

Theorem 1. A sub-F function has a unique support at all but a countable set of points.

First consider two points $x_{0}$ and $x_{1}$ at which the supports are not unique, and at which the extreme supports are $F_{0^{+}}^{+} F_{\overline{0}}^{-}$, and $F_{1}^{+}, F_{1}^{-}$, respectively. Let $A_{0}$ be the open set of points to the right of $x_{0}$ and between $F_{0}^{+}$and $F_{0}^{-}$; similarly define $A_{1}$. Then $A_{0}$ and $A_{1}$ do not intersect. For suppose that $\left(x_{2}, y_{2}\right)$ is a common point. The $F$-line $F^{*}$ determined by $\left(x_{2}, y_{2}\right)$ and $\left(x_{0}, f\left(x_{0}\right)\right)$ supports $f$ at $x_{0}$, by P1. By Lemma $1, F^{*} \leqq F_{1}^{-}$for $x>x_{1}$, and in particular, $F^{*}\left(x_{2}\right)=y_{2} \leqq F_{1}^{-}\left(x_{2}\right)$. This makes it impossible for $\left(x_{2}, y_{2}\right)$ to be in $A_{1}$.

To each point $x$ at which the support to $f$ is not unique we assign the open set $A$ to the right of $x$ and between the extreme supports. By 
the preceding argument these open sets are disjoint and hence countable. This completes the proof.

THEOREM 2. Let $\left\{f_{n}\right\}$ be a sequence of sub-F functions converging to a limit function $f$. Then $f$ is sub- $F$, and the convergence is uniform in every compact subinterval of $I$.

The $F$-line determined by $\left(x_{i}, f\left(x_{i}\right)\right)$ and $\left(x_{j}, f\left(x_{j}\right)\right)$ we denote by $F_{i j}$; similarly for $f_{n}$. Let $x_{0}<x_{1}<x_{2}$. Then $f_{n}\left(x_{1}\right) \leqq F_{n, 02}\left(x_{1}\right)$. By B2, $\lim F_{n, 02}=F_{02}$; thus $\lim f_{n}\left(x_{1}\right)=f\left(x_{1}\right) \leqq \lim F_{n, 02}\left(x_{1}\right)=F_{02}\left(x_{1}\right)$, and $f$ is sub- $F$.

Now suppose that the convergence is not uniform. There exists a sequence $\left\{x_{n}\right\}$ tending to a point $x_{0}$ of $I$ such that $\left|f_{n}\left(x_{n}\right)-f\left(x_{n}\right)\right|$ $>\epsilon>0$. We may assume that $x_{n}>x_{0}$ without loss of generality. By choosing suitable subsequences, we can assume that either (a) $f_{n}\left(x_{n}\right)$ $<f\left(x_{n}\right)-\epsilon$, or (b) $f_{n}\left(x_{n}\right)>f\left(x_{n}\right)+\epsilon$. First take case (a). Let $x^{*}<x_{0}$ and $F^{*}$ be the $F$-line determined by $\left(x^{*}, f\left(x^{*}\right)+\epsilon / 2\right)$ and $\left(x_{0}, f\left(x_{0}\right)-\epsilon / 2\right)$. The functions $f$ and $F^{*}$ are continuous, and by choosing $n$ sufficiently large, we can assure that $\left|f\left(x_{n}\right)-f\left(x_{0}\right)\right|<\epsilon / 4$ and also that $\mid F^{*}\left(x_{n}\right)$ $-F^{*}\left(x_{0}\right) \mid<\epsilon / 4$. Then

$$
f_{n}\left(x_{n}\right)<f\left(x_{n}\right)-\epsilon<f\left(x_{0}\right)-3 \epsilon / 4=F^{*}\left(x_{0}-\epsilon / 4<F^{*}\left(x_{n}\right) .\right.
$$

Also let $n$ be so great that $f_{n}\left(x^{*}\right)<f\left(x^{*}\right)+\epsilon / 2=F^{*}\left(x^{*}\right)$. Then $F^{*}>f_{n}$ at $x^{*}$ and $x_{n}$, and since $f$ is sub- $F$, we have $f_{n}\left(x_{0}\right)<F^{*}\left(x_{0}\right)$. Letting $n \rightarrow \infty$, it follows that $f\left(x_{0}\right)=\lim f_{n}\left(x_{0}\right) \leqq F^{*}\left(x_{0}\right)=f\left(x_{0}\right)-\epsilon / 2$, an impossibility. Thus (a) is not possible. If case (b) holds, let $x^{*}>x_{0}$ and $F_{n}^{*}$ be determined by $f_{n}$ at $x_{0}$ and $x^{*}$, and $F^{*}$ by $f$ at the same points. Then $f_{n}\left(x_{n}\right) \leqq F_{n}^{*}\left(x_{n}\right)$. Now $F_{n}^{*}(x) \rightarrow F^{*}(x)$ uniformly in an interval containing $x_{0}$, and so $F_{n}^{*}\left(x_{n}\right) \rightarrow F^{*}\left(x_{0}\right)=f\left(x_{0}\right)$. This says that $\lim \sup f_{n}\left(x_{n}\right) \leqq f\left(x_{0}\right)$, which contradicts (b), and the theorem is proved.

3. Differentiability. Beckenbach points out that the function $F$ is itself sub- $F$ and constructs a family $\mathcal{F}$ all of whose members are nowhere differentiable. A sub- $F$ function thus need not be differentiable unless further conditions are placed on $\mathcal{F}$. We now place two such conditions.

A. Each F-line shall be continuously differentiable.

B. If $F_{n} \rightarrow F$, then $F_{n}^{\prime} \rightarrow F^{\prime}$, uniformly in every compact subinterval of $I$.

Henceforth $\mathcal{F}$ will be assumed to satisfy $\mathrm{A}$ and $\mathrm{B}$, and the sub- $F$ functions will be said to be sub-FS.

Lemma 2. Let $J$ be a compact subinterval of $I$, and $\delta$ and $M$ two 
positive numbers. There exists a function $B(J, \delta, M)$ such that if $x, x_{1}$, and $x_{2}$ are in $J,\left|x_{1}-x_{2}\right|>\delta,\left|y_{1}\right|<M,\left|y_{2}\right|<M$, then $\left|F_{12}^{\prime}(x)\right|$ $<B(J, \delta, M)$.

The proof follows straightforwardly from $A$ and $B$ and simple compactness considerations, and will be omitted. One might imagine that B follows from A much in the same manner that Beckenbach was able to prove B2 from the continuity and uniqueness of the $F$. This is not the case, however. It is easy to give examples to show that even A and Lemma 2 do not imply B; in fact, no amount of smoothness of the individual curves will imply $B$. Theorems 3 and 4 below are proved on the basis of $A$ and Lemma 2, but the other results also use B.

Theorem 3. A sub-FS function satisfies a Lipschitz condition in every compact subinterval $J$ of $I$, and thus is absolutely continuous and has a derivative almost everywhere, which is bounded in $J$.

If $J$ is the interval $a^{\prime} \leqq x \leqq b^{\prime}$, we consider the interval $J_{1}: a^{\prime}-\delta$ $\leqq x \leqq b^{\prime}+\delta$, where $\delta>0$ is sufficiently small so that $J_{1} \subset I$. Let $M$ be the maximum of $|f|$ in $J_{1}$, and let $F^{+}$be determined by $\left(x_{0}, f\left(x_{0}\right)\right)$ and $\left(b^{\prime}+\delta, f\left(b^{\prime}+\delta\right)\right)$ and $F^{-}$by $\left(x_{0}, f\left(x_{0}\right)\right)$ and $\left(a^{\prime}-\delta, f\left(a^{\prime}-\delta\right)\right)$. If $x_{1} \in J$, we see from $\mathrm{B} 3$ that

$$
\begin{aligned}
& F^{\prime \prime}\left(\bar{x}_{1}\right)=\frac{F^{\sim}\left(x_{1}\right)-F^{\llcorner}\left(x_{0}\right)}{x_{1}-x_{0}} \leqq \frac{f\left(x_{1}\right)-f\left(x_{0}\right)}{x_{1}-x_{0}} \leqq \frac{F^{+}\left(x_{1}\right)-F^{+}\left(x_{0}\right)}{x_{1}-x_{0}} \\
& =F^{+^{\prime}}\left(\stackrel{*}{x_{1}}\right) \text {, }
\end{aligned}
$$

where $\bar{x}_{1}$ and $x_{1}^{*}$ are between $x_{0}$ and $x_{1}$, and hence are in $J$. By Lemma 2 it follows that $|\Delta f / \Delta x|<B\left(J_{1}, \delta, M\right)$, and the theorem is proved.

TheOREM 4. If $\lim f_{n}=f$, where $f_{n}$ is sub-FS, then the $f_{n}$ satisfy a uniform Lipschitz condition in any compact subinterval of $I$, and thus the derivatives $f_{n}^{\prime}$ are uniformly bounded.

The proof is just like that of Theorem 3, with the added remark that the $f_{n}$ have a common bound.

TheOREM 5. If $f$ is sub-FS and has a unique support $F_{0}$ at $x_{0}$, then $f^{\prime}\left(x_{0}\right)$ exists and equals $F_{0}^{\prime}\left(x_{0}\right)$.

Let $x_{1}>x_{0}$, and consider $F_{01}(x)$. We have

$$
\frac{f\left(x_{1}\right)-f\left(x_{0}\right)}{x_{1}-x_{0}}=\frac{F_{01}\left(x_{1}\right)-F_{01}\left(x_{0}\right)}{x_{1}-x_{0}}=F_{01}^{\prime}\left(\bar{x}_{1}\right),
$$

where $x_{0}<\bar{x}_{1}<x_{1}$. As $x_{1} \rightarrow x_{0}$, we see as in $\mathrm{P} 1$ that $F_{01}(x)$ tends to 
$F_{0}(x)$, the unique support to $f$ at $x_{0}$. By hypothesis $\mathrm{B}, F_{01}^{\prime}(x) \rightarrow F_{0}^{\prime}(x)$, uniformly in an interval containing $x_{0}$. Since $\bar{x}_{1} \rightarrow x_{0}$, we have that $F_{01}^{\prime}\left(\bar{x}_{1}\right) \rightarrow F_{0}^{\prime}\left(x_{0}\right)$, and $f$ has its right-hand derivative equal to $F_{0}^{\prime}\left(x_{0}\right)$. Similarly for the left-hand derivative and the theorem is proved. Actually one sees that we have proved more than the theorem stated; namely that the right- and left-hand derivatives always exist and equal the slopes of the extreme supports at $x_{0}$.

The converse of Theorem 5 is not true: $f^{\prime}$ may exist without there being a unique support. An example of this is given by the family $\mathcal{F}: y=(a x+b)^{3}$. The function $|x|^{8}$ is sub-FS and has a derivative and many supports at the origin.

Theorem 6. $A$ sub-FS function has a derivative at all but a countable set of points.

This is a consequence of Theorems 1 and 5 .

REMARK. The hypotheses under which we showed that $f$ has a derivative almost everywhere are more restrictive than Peixoto's, but the result is stronger. Our hypotheses for proving Theorem 6 neither imply nor are implied by those of Peixoto for P3. On the other hand they are such as to permit us to show the connection between absence of derivative and non-unique support.

THEOREM 7. Let $\left\{f_{n}\right\}$ be a sequence of sub-FS functions with limit $f$. Then with the exception of a countable set, $\lim f_{n}^{\prime}(x)=f^{\prime}(x)$.

Let $x_{0}$ be a point at which $f$ and all $f_{n}$ have unique supports. There are only countably many exceptions. Let $x_{1}>x_{0}$ and let $F_{n, 0}$ be the support to $f_{n}$ at $x_{0}$. Then for $x>x_{0}, F_{n, 01}(x) \geqq F_{n, 0}(x)$, and so $F_{n, 01}^{\prime}\left(x_{0}\right)$ $\geqq F_{n, 0}^{\prime}\left(x_{0}\right)=f_{n}^{\prime}\left(x_{0}\right)$. Operating similarly with $x_{2}<x_{0}$, we find

$$
F_{n, 20}^{\prime}\left(x_{0}\right) \leqq f_{n}^{\prime}\left(x_{0}\right) \leqq F_{n, 01}^{\prime}\left(x_{0}\right) \text {. }
$$

As $n \rightarrow \infty, F_{n, 20} \rightarrow F_{20}$ and $F_{n, 01} \rightarrow F_{01}$, and by B,

$$
F_{20}^{\prime}\left(x_{0}\right) \leqq \lim \inf f_{n}^{\prime}\left(x_{0}\right) \leqq \lim \sup f_{n}^{\prime}\left(x_{0}\right) \leqq F_{01}^{\prime}\left(x_{0}\right) .
$$

Now let $x_{1} \rightarrow x_{0}$ and $x_{2} \rightarrow x_{0}$. As in P1, $F_{20} \rightarrow F_{0}$ and $F_{01} \rightarrow F_{0}$, and by B, $F_{20}^{\prime}\left(x_{0}\right) \rightarrow F_{0}^{\prime}\left(x_{0}\right), F_{01}^{\prime}\left(x_{0}\right) \rightarrow F_{0}^{\prime}\left(x_{0}\right)$. Thus $\lim f_{n}^{\prime}\left(x_{0}\right)=F_{0}^{\prime}\left(x_{0}\right)=f^{\prime}\left(x_{0}\right)$.

4. An application. A function is called sub-sine if in every interval of length less than $2 \pi$ it is sub- $F$, with $\mathcal{F}$ the family: $a \cos \theta+b \sin \theta$. $\mathcal{F}$ clearly satisfies conditions $A$ and $B$. The sub-sine functions $p(\theta)$ with period $2 \pi$ are the supporting functions of convex curves [4]. If $p$ is sufficiently regular, the formula for the area $S$ of the convex curve represented by $p$ is $S=(1 / 2) \int_{0}^{2 \pi}\left(p^{2}-p^{\prime 2}\right) d \theta[2$, p. 58]. Any 
supporting function $p$ is the limit of analytic ones, $p_{n}[2$, p. 36]. Because of the periodicity of $p$ we see by Theorems 2,4 , and 7 that $p_{n} \rightarrow p$ uniformly and $p_{n}^{\prime} \rightarrow p^{\prime}$ boundedly for all $\theta$; thus $S=\lim S_{n}$ $=\lim (1 / 2) \int_{0}^{2 \pi}\left(p_{n}^{2}-p_{n}^{\prime 2}\right) d \theta=(1 / 2) \int_{0}^{2 \pi}\left(p^{2}-p^{\prime 2}\right) d \theta$, and we have a simple proof of the known fact that the integral $(1 / 2) \int_{0}^{2 \pi}\left(p^{2}-p^{\prime 2}\right) d \theta$ represents the area for the general convex curve.

\section{REFERENCES}

1. E. F. Beckenbach, Generalized convex functions, Bull. Amer. Math. Soc. vol. 43 (1937) pp. 363-371.

2. T. Bonnesen and W. Fenchel, Theorie der Konvexen Körper, Berlin, Springer, 1934.

3. F. F. Bonsall, The characterization of generalized convex functions, Quart. J, Math. Oxford Ser. (2) vol. 1 (1950) pp. 100-111.

4. J. W. Green, Sets subtending a constant angle on a circle, Duke Math. J. vol. 17 (1950) pp. 263-267.

5. M. Matos Peixoto, On the existence of derivatives of generalized convex functions, Summa Brasil. Math. vol. 2 (1948) pp. 35-42.

6. - Convexity of curves, Notas de Matematica, no. 6, Rio de Janiero, Boffoni, 1948.

7. - Generalized convex functions and second order differential inequalities, Bull. Amer. Math. Soc. vol. 55 (1949) pp. 563-572.

The University of California, Los Angeles, and The Institute for Advanced Study 